\title{
LINEAR MAPS PRESERVING RANK 2 ON THE SPACE OF ALTERNATE MATRICES AND THEIR APPLICATIONS
}

\author{
CHONGGUANG CAO and XIAOMIN TANG
}

Received 18 January 2004

\begin{abstract}
Denote by $\mathscr{K}_{n}(\mathbf{F})$ the linear space of all $n \times n$ alternate matrices over a field $\mathbf{F}$. We first characterize all linear bijective maps on $\mathscr{K}_{n}(\mathbf{F})(n \geq 4)$ preserving rank 2 when $\mathbf{F}$ is any field, and thereby the characterization of all linear bijective maps on $\mathscr{K}_{n}(\mathbf{F})$ preserving the max-rank is done when $\mathbf{F}$ is any field except for $\{0,1\}$. Furthermore, the linear preservers of the determinant (resp., adjoint) on $\mathscr{K}_{n}(\mathbf{F})$ are also characterized by reducing them to the linear preservers of the max-rank when $n$ is even and $\mathbf{F}$ is any field except for $\{0,1\}$. This paper can be viewed as a supplement version of several related results.
\end{abstract}

2000 Mathematics Subject Classification: 15A03, 15A04.

1. Introduction. Let $\mathbf{F}$ be any field and $\mathbf{F}^{*}$ its multiplicative group. Denote by $M_{m \times n}(\mathbf{F})$ the space of all $m \times n$ matrices over $\mathbf{F}$ and by GL $\mathrm{G}_{n}(\mathbf{F})$ the subset of $M_{n \times n}(\mathbf{F})$ consisting of all invertible matrices. The notation $E_{i j}$ denotes the matrix with 1 at the $(i, j)$ th entry and 0 elsewhere. For $A \in M_{m \times n}(\mathbf{F})$, we denote by $A^{t}$ the transpose of $A$. A square matrix $A$ is said to be alternate if $A^{t}=-A$ and all diagonal elements are zeros. By [2, page 161], we have the following.

(i) When the characteristic of $\mathbf{F}$ is not 2, a matrix is alternate if and only if it is skew symmetric; when the characteristic of $\mathbf{F}$ is 2, a matrix is alternate if and only if it is symmetric and all diagonal elements are zeros.

(ii) The rank of any alternate matrix is necessarily even.

(iii) $A$ is an alternate matrix of $\operatorname{rank} A=2 r>0$ if and only if there exists a matrix $P \in \mathrm{GL}_{n}(\mathbf{F})$ such that $P^{t} A P=\sum_{i=1}^{r} J_{i}^{(n)}$, where $J_{i}^{(n)}=E_{2 i-1} 2 i-E_{2 i 2 i-1} \in \mathcal{K}_{n}(F)$.

(iv) Under the usual addition and scalar multiplication, the set $\mathscr{K}_{n}(\mathbf{F})$ of all alternate matrices in $M_{n \times n}(\mathbf{F})$ forms a linear space over $\mathbf{F}$.

A matrix $A \in \mathscr{K}_{n}(\mathbf{F})$ is said to have max-rank if $\operatorname{rank} A=n$ (resp., $n-1$ ) when $n$ is even (resp., odd). Denote by $M K_{n}(\mathbf{F})$ the subset of $\mathscr{K}_{n}(\mathbf{F})$ consisting of all max-rank matrices. For $A \in \mathscr{K}_{n}(\mathbf{F})$, the notation $A^{\text {ad }}$ denotes the adjoint matrix of $A$, that is, $A^{\text {ad }}=B=\left[b_{i j}\right]$, where $b_{j i}$ is the cofactor of the $i, j$ entry of $A$.

A linear map $\phi: \mathscr{K}_{n}(\mathbf{F}) \rightarrow \mathscr{K}_{n}(\mathbf{F})$ is said to preserve rank 2 if $\operatorname{rank} \phi(A)=2$ for all $A \in \mathscr{K}_{n}(\mathbf{F})$ with $\operatorname{rank} A=2$. A linear map $\phi: \mathscr{K}_{n}(\mathbf{F}) \rightarrow \mathscr{K}_{n}(\mathbf{F})$ is said to preserve the max-rank if $\phi\left(M K_{n}(\mathbf{F})\right)=\mathcal{M} K_{n}(\mathbf{F})$. A linear map $\phi: \mathscr{K}_{n}(\mathbf{F}) \rightarrow \mathscr{K}_{n}(\mathbf{F})$ is said to preserve the determinant (resp., the adjoint) if $\operatorname{det} \phi(A)=\operatorname{det} A\left(\operatorname{resp} ., \phi(A)^{\mathrm{ad}}=\phi\left(A^{\mathrm{ad}}\right)\right)$ for all $A \in \mathscr{K}_{n}(\mathbf{F})$.

Linear preserver problems are an active research area in matrix theory (see $[3,5]$ and the references therein). The category of linear rank 1 preserver problems on $M_{m \times n}(\mathbf{F})$ is 
very important since it can be used to solve the other linear preserver problems. Since alternate matrix plays an important role in the theory of quadratic forms and classical groups (see [7]), it is worthwhile and interesting to study the preserving problems of alternate matrices. However, one can not study linear rank 1 preserver problems on $\mathscr{K}_{n}(\mathbf{F})$ since the rank of any matrix in $\mathscr{K}_{n}(\mathbf{F})$ is even.

To give a parallel result to those of linear rank 1 preserver problems on $M_{m \times n}(\mathbf{F})$, this paper first characterizes all linear bijective maps on $\mathscr{K}_{n}(\mathbf{F})$ preserving rank 2 when $n \geq 4$ and $\mathbf{F}$ is any field (see Section 2), and then all linear bijective maps on $\mathscr{K}_{n}(\mathbf{F})$ preserving the max-rank are characterized by using the result about rank 2 preservers when $n \geq 4$ and $\mathbf{F}$ is any field except for $\{0,1\}$ (see Section 3). Furthermore, the linear preservers of the determinant (resp., the adjoint) on $\mathscr{K}_{n}(\mathbf{F})$ are also characterized by reducing them to the linear preservers of the max-rank when $n \geq 4$ is even and $\mathbf{F}$ is any field except for $\{0,1\}$ (see Section 4 ).

We provide some notations which will be used in the rest of this paper. We denote by $I_{k}$ and $O_{m \times n}$ the $k \times k$ identity matrix and the $m \times n$ zero matrix, respectively. We also write them as $I$ and $O$, respectively, when the dimensions of these matrices are clear. Let $J=\left[\begin{array}{cc}0 & 1 \\ -1 & 0\end{array}\right]$. Clearly, $J$ is the same as $J_{1}^{(2)}$.

The basic tool used in this paper is the following fundamental theorem of geometry of alternate matrices.

THEOREM 1.1 (see [7]). Let $\mathbf{F}$ be any field, $n \geq 4$ an integer, and $\phi$ a bijective map from $\mathscr{K}_{n}(\mathbf{F})$ to itself. Assume that for any $X_{1}, X_{2} \in \mathscr{K}_{n}(\mathbf{F}), \operatorname{rank}\left(X_{1}-X_{2}\right)=2$ if and only if $\operatorname{rank}\left(\phi\left(X_{1}\right)-\phi\left(X_{2}\right)\right)=2$. Then, there exist $a \in \mathbf{F}^{*}, Q \in \mathrm{GL}_{n}(\mathbf{F}), K_{0} \in \mathcal{K}_{n}(\mathbf{F})$, and an automorphism $\sigma$ on $\mathbf{F}$ such that either

$$
\phi(X)=a Q^{t} X^{\sigma} Q+K_{0} \quad \forall X=\left[x_{i j}\right] \in \mathscr{K}_{n}(\mathbf{F})
$$

or

$$
n=4, \quad \phi(X)=a Q^{t}\left(X^{*}\right)^{\sigma} Q+K_{0} \quad \forall X=\left[x_{i j}\right] \in \mathscr{K}_{4}(\mathbf{F}),
$$

where $X^{\sigma}=\left[\sigma\left(x_{i j}\right)\right]$ and

$$
X^{*}=\left[\begin{array}{cccc}
0 & x_{12} & x_{13} & x_{23} \\
-x_{12} & 0 & x_{14} & x_{24} \\
-x_{13} & -x_{14} & 0 & x_{34} \\
-x_{23} & -x_{24} & -x_{34} & 0
\end{array}\right]
$$

Conversely, any map of the form (1.1) and (1.2) from $\mathcal{K}_{n}(\mathbf{F})$ to itself is bijective and both the map and its inverse preserve the adjacency (recall that $X_{1}$ and $X_{2}$ are adjacent if $\operatorname{rank}\left(X_{1}-X_{2}\right)=2$ for $X_{1}, X_{2} \in \mathscr{K}_{n}(\mathbf{F})$ ).

2. Linear maps preserving rank 2 . In this section, we assume that $\mathbf{F}$ is any field and $n \geq 4$ is an integer. Denote by $e_{k}^{(n)}$ the $n$-dimensional column vector with 1 at the $k$ th 
entry and 0 elsewhere. Further, for arbitrary but fixed $W \in \mathrm{GL}_{n}(\mathbf{F})$, denote

$$
\begin{gathered}
\Theta_{W}=\left\{W^{t} \operatorname{diag}\left(X_{11}, O\right) W \mid X_{11} \in \mathcal{K}_{n-2}(\mathbf{F})\right\}, \\
\Omega_{W}=\left\{W^{t}\left[\begin{array}{cc}
X_{11} & X_{12} \\
-X_{12}^{t} & O
\end{array}\right] W \mid X_{11} \in \mathcal{K}_{n-2}(\mathbf{F}), X_{12} \in M_{(n-2) \times 2}(\mathbf{F})\right\} .
\end{gathered}
$$

LEMMA 2.1. Given $A \in \mathcal{K}_{n}(\mathbf{F})$ with $\operatorname{rank} A=2$ and $W \in \mathrm{GL}_{n}(\mathbf{F})$. Further, assume that there exists nonzero $B \in \Theta_{W}$ satisfying $\operatorname{rank}(A+B)=2$. Then, $A \in \Omega_{W}$.

Proof. Let $A=W^{t}\left[\begin{array}{cc}A_{1} & A_{2} \\ -A_{2}^{t} & A_{3}\end{array}\right] W$ with $A_{3} \in \mathscr{K}_{2}(\mathbf{F})$. If $A_{3} \neq O$, then $A_{3}$ is invertible, that is, $\operatorname{rank} A_{3}=2$. Let $P=\left[\begin{array}{cc}I_{n-2} & O \\ -A_{3}^{-1} A_{2}^{t} I_{2}\end{array}\right] W$. Then, $B=P^{t} B_{1} P$ for some $B_{1} \in \Theta_{W}$ and $A=P^{t} \operatorname{diag}\left(A_{1}+A_{2} A_{3}^{-1} A_{2}^{t}, A_{3}\right) P$. This, together with $\operatorname{rank} A=\operatorname{rank}(A+B)=\operatorname{rank} A_{3}=2$, yields $B_{1}=O$, and hence $B=O$, an impossibility. Therefore, $A_{3}=O$. The proof is complete.

LEMMA 2.2. Let $\phi: \mathscr{K}_{n}(\mathbf{F}) \rightarrow \mathscr{K}_{n}(\mathbf{F})$ be a linear map preserving rank 2 and satisfying

$$
\phi\left(J_{k}^{(n)}\right) \in \Theta_{W} \quad \text { for some } 1 \leq k \leq\left[\frac{n}{2}\right] .
$$

Then, $\phi\left(S_{1}(k) \cup S_{2}(k)\right) \subset \Omega_{W}$ with $S_{1}(k)=\left\{E_{i j}-E_{j i} \mid j>i, i \in\{2 k-1,2 k\}\right\}$, and $S_{2}(k)=$ $\left\{E_{i j}-E_{j i} \mid i<j, j \in\{2 k-1,2 k\}\right\}$.

Proof. For arbitrary but fixed $A_{i j}=E_{i j}-E_{j i} \in S_{1}(k) \cup S_{2}(k)$, it is clear that $\operatorname{rank} A_{i j}=$ $\operatorname{rank} J_{k}^{(n)}=\operatorname{rank}\left(J_{k}^{(n)}+A_{i j}\right)=2$. By the definition of $\phi$, we have $\operatorname{rank} \phi\left(A_{i j}\right)=$ $\operatorname{rank} \phi\left(J_{k}^{(n)}\right)=\operatorname{rank}\left(\phi\left(J_{k}^{(n)}\right)+\phi\left(A_{i j}\right)\right)=2$. Using (2.2) and applying Lemma 2.1 to $A=\phi\left(A_{i j}\right)$ and $B=\phi\left(J_{k}^{(n)}\right)$ yield $\phi\left(A_{i j}\right) \in \Omega_{W}$. Thus, the proof is complete.

LEMMA 2.3. Let $\phi: \mathscr{K}_{n}(\mathbf{F}) \rightarrow \mathscr{K}_{n}(\mathbf{F})$ be a linear bijective map preserving rank 2 . Then, there exists a full-row rank matrix $P \in M_{2[n / 2] \times n}(\mathbf{F})$ such that $\phi\left(J_{i}^{(n)}\right)=P^{t} J_{i}^{(2[n / 2])} P$ for all $1 \leq i \leq[n / 2]$.

Proof. For any $1 \leq i \leq[n / 2]$, it follows from $\operatorname{rank} J_{i}^{(n)}=2$ that $\operatorname{rank} \phi\left(J_{i}^{(n)}\right)=2$. Thus, we can write

$$
\phi\left(J_{i}^{(n)}\right)=P_{i}^{t} J P_{i}, \quad i=1, \ldots,\left[\frac{n}{2}\right],
$$

where $P_{i} \in M_{2 \times n}(\mathbf{F})$ satisfies $\operatorname{rank} P_{i}=2$ for all $1 \leq i \leq[n / 2]$. Let

$$
P=\left[\begin{array}{c}
P_{1} \\
\vdots \\
P_{[n / 2]}
\end{array}\right], \quad Z_{i}=\left[\begin{array}{ll}
e_{2 i-1}^{(2[n / 2])} & e_{2 i}^{(2[n / 2])}
\end{array}\right]^{t}, \quad i=1, \ldots,\left[\frac{n}{2}\right] .
$$

Then, $P_{i}=Z_{i} P$ and $Z_{i}^{t} J Z_{i}=J_{i}^{(2[n / 2])}$. This, together with (2.3), gives

$$
\phi\left(J_{i}^{(n)}\right)=P^{t} J_{i}^{(2[n / 2])} P, \quad i=1, \ldots,\left[\frac{n}{2}\right] .
$$

Now, to complete the proof, it suffices to show that $\operatorname{rank} P=s=2[n / 2]$. This is proceeded by the reduction to absurdity. Suppose $2 \leq s \leq 2[n / 2]-1$. Then, there exists 
a matrix $W \in \mathrm{GL}_{n}(\mathbf{F})$ satisfying $P=\left[P_{0} O\right] W$, where $P_{0} \in M_{2[n / 2] \times s}(\mathbf{F})$ with $\operatorname{rank} P_{0}=s$. Substituting $P$ into (2.5) yields

$$
\phi\left(J_{i}^{(n)}\right)=W^{t} \operatorname{diag}\left(P_{0}^{t} J_{i}^{(2[n / 2])} P_{0}, O\right) W, \quad i=1, \ldots,\left[\frac{n}{2}\right] .
$$

If $s \leq n-2$, then, by (2.6), we have $\phi\left(J_{k}^{(n)}\right) \in \Theta_{W}$ for all $1 \leq k \leq[n / 2]$. Using Lemma 2.2 yields $\phi\left(\left\{E_{p q}-E_{q p} \mid 1 \leq p<q \leq n\right\}\right) \subset \Omega_{W}$. Noting that any matrix in $\mathscr{K}_{n}(\mathbf{F})$ can be written as a linear combination of finite matrices in the set $\left\{E_{p q}-E_{q p} \mid 1 \leq p<q \leq n\right\}$, we deduce from the linearity of $\phi$ that $\phi\left(\mathscr{K}_{n}(\mathbf{F})\right) \subset \Omega_{W}$, which contradicts the surjectivity of $\phi$. Therefore, $s \geq n-1$. This, together with $2 \leq s \leq 2[n / 2]-1$, implies that $n=2[n / 2]$ and $s=2[n / 2]-1=n-1$. Furthermore, the matrix $P_{0}$ can be written as $P_{0}=Z R_{1}$ with

$$
Z=\left[\begin{array}{lllllll}
e_{1}^{(n-1)} & \cdots & e_{v-1}^{(n-1)} & z & e_{v}^{(n-1)} & \cdots & e_{n-1}^{(n-1)}
\end{array}\right]^{t}
$$

where $z \in M_{(n-1) \times 1}(\mathbf{F})$ and $R_{1} \in \mathrm{GL}_{n-1}(\mathbf{F})$. Let $R=\left(R_{1} \oplus 1\right) W$. Then, (2.6) turns into

$$
\phi\left(J_{i}^{(n)}\right)=R^{t} \operatorname{diag}\left(Z^{t} J_{i}^{(n)} Z, O\right) R, \quad i=1, \ldots,\left[\frac{n}{2}\right]
$$

Without loss of generality, we can assume that $v=n$, or equivalently,

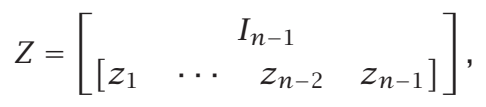

where $z_{i} \in \mathbf{F}$ for all $1 \leq i \leq n-1$. (Otherwise, the rest of the proof can be proceeded by denoting a new set which is similar to $\Omega_{W}$.) By direct computation, then (2.8) implies

$$
\begin{aligned}
\phi\left(J_{i}^{(n)}\right) & =R^{t} J_{i}^{(n)}, \quad R \in \Theta_{R}, i=1,2, \ldots,\left[\frac{n}{2}\right]-1, \\
\phi\left(J_{[n / 2]}^{(n)}\right) & =R^{t}\left[\begin{array}{ccccc}
O_{n-2} & -\left[z_{1}\right. & \ldots & \left.z_{n-2}\right]^{t} & O \\
{\left[z_{1}\right.} & \ldots & z_{n-2} & 0 & 0 \\
O & 0 & 0 &
\end{array}\right], \quad R \in \Omega_{R} .
\end{aligned}
$$

This, together with Lemma 2.2, gives $\phi\left(\left\{E_{p q}-E_{q p} \mid 1 \leq p<q \leq n\right\}\right) \subset \Omega_{R}$. Noting that any matrix in $\mathscr{K}_{n}(\mathbf{F})$ can be written as a linear combination of finite matrices in the set $\left\{E_{p q}-E_{q p} \mid 1 \leq p<q \leq n\right\}$, we deduce from the linearity of $\phi$ that $\phi\left(\mathscr{K}_{n}(\mathbf{F})\right) \subset \Omega_{R}$, which contradicts the surjectivity of $\phi$. In summary, $s=2[n / 2]$.

The proof is complete.

THEOREM 2.4. Suppose $F$ is any field and $n \geq 4$ is an integer. Then, $\phi: \mathscr{K}_{n}(\mathbf{F}) \rightarrow \mathscr{K}_{n}(\mathbf{F})$ is a linear bijective map preserving rank 2 if and only if there exist $\delta \in \mathbf{F}^{*}$ and $Q \in \mathrm{GL}_{n}(\mathbf{F})$ satisfying either

$$
\phi(X)=\delta Q^{t} X Q \quad \forall X \in \mathscr{K}_{n}(\mathbf{F})
$$


or

$$
n=4, \quad \phi(X)=\delta P^{t} X^{*} P \quad \forall X \in \mathscr{K}_{n}(\mathbf{F}),
$$

where $X^{*}$ is defined in (1.3).

Proof. The "if" part is obvious. Now we prove the "only if" part. For arbitrary but fixed nonzero $A \in \mathscr{K}_{n}(\mathbf{F})$, let $\operatorname{rank} A=2 r>0$. It is clear that there exists $W \in \mathrm{GL}_{n}(\mathbf{F})$ such that $A=W^{t}\left(\sum_{i=1}^{r} J_{i}^{(n)}\right) W$. Denote a new map $\psi: \mathscr{K}_{n}(\mathbf{F}) \rightarrow \mathscr{K}_{n}(\mathbf{F})$ by $\psi(Z)=$ $\phi\left(W^{t} Z W\right)$ for all $Z \in \mathscr{K}_{n}(\mathbf{F})$. Then, $\psi$ is also a linear bijective map preserving rank 2. By Lemma 2.3, there exists a full-row rank matrix $P \in M_{2[n / 2] \times n}(\mathbf{F})$ such that $\psi\left(\sum_{i=1}^{r} J_{i}^{(n)}\right)=$ $P^{t}\left(\sum_{i=1}^{r} J_{i}^{(2[n / 2])}\right) P$, and hence $\phi(A)=P^{t}\left(\sum_{i=1}^{r} J_{i}^{(2[n / 2])}\right) P$. This implies that $\operatorname{rank} \phi(A)=$ $\operatorname{rank} A$ for all nonzero $A \in \mathscr{K}_{n}(\mathbf{F})$. Using the linearity and nonsingularity of $\phi$ yields that, for any $X_{1}, X_{2} \in \mathscr{K}_{n}(\mathbf{F}), \operatorname{rank}\left(X_{1}-X_{2}\right)=2$ if and only if $\operatorname{rank}\left(\phi\left(X_{1}\right)-\phi\left(X_{2}\right)\right)=2$. By Theorem 1.1, $\phi$ has one of the forms (1.1) or (1.2). Again, using the linearity of $\phi$ completes the proof.

\section{Linear maps preserving the max-rank}

LEMmA 3.1. Suppose $F$ is any field and $n \geq 4$ is an integer. Let $X, Y \in \mathscr{K}_{n}(\mathbf{F})$ with $\operatorname{rank} X=2$. Then, there exist matrices $G \in \mathscr{K}_{2}(\mathbf{F})$ and $P \in \mathrm{GL}_{n}(\mathbf{F})$ such that

$$
P^{t} X P=J_{1}^{(n)}, \quad P^{t} Y P=\left[\begin{array}{ccc}
G & O & H \\
O & D_{1} & O \\
-H^{t} & O & O_{r \times r}
\end{array}\right],
$$

where $r$ is a nonnegative integer and either $D_{1} \in \mathscr{K}_{n-2-r}(\mathbf{F})$ is nonsingular or $D_{1}$ vanishes.

Proof. Without loss of generality, we can assume that $X=J_{1}^{(n)}$. Let $Y=\left[\begin{array}{cc}E & C \\ -C^{t} & D\end{array}\right]$, where $E \in \mathscr{K}_{2}(\mathbf{F})$. If $D=O$, then the proof is complete by letting $P=I, G=E$, and $H=C$. If $D \neq O$, by (iii) in Section 1 , we know that there exist matrices $D_{1} \in \mathscr{K}_{n-2-r}(\mathbf{F}) \cap$ $\mathrm{GL}_{n-2-r}(\mathbf{F})$ and $P_{1} \in \mathrm{GL}_{n-2}(\mathbf{F})$ such that $P_{1}^{t} D P_{1}=\operatorname{diag}\left(D_{1}, O\right)$. Let $P_{2}=\operatorname{diag}\left(I_{2}, P_{1}\right)$. Then,

$$
P_{2}^{t} X P_{2}=J_{1}^{(n)}, \quad P_{2}^{t} Y P_{2}=\left[\begin{array}{ccc}
E & C_{1} & H \\
-C_{1}^{t} & D_{1} & O \\
-H^{t} & O & O_{r \times r}
\end{array}\right],
$$

where $\left[C_{1} H\right]=C P_{1}$. Denote

$$
P=P_{2}\left[\begin{array}{ccc}
I_{2} & O & O \\
D_{1}^{-1} C_{1}^{t} & I & O \\
O & O & I_{r}
\end{array}\right]
$$

and $G=E+C_{1} D_{1}^{-1} C_{1}^{t}$. Then, by (3.2), the proof is complete. 
LEMMA 3.2. Suppose $\mathbf{F}$ is any field and $n \geq 4$ is an integer, $P^{t} Y P$ is of the form (3.1), where $P$ is in $\mathrm{GL}_{n}(\mathbf{F})$ and $Y$ is in $\mathcal{K}_{n}(\mathbf{F})$. Then, $Y \in M K_{n}(\mathbf{F})$ if and only if one of the following three conclusions holds:

(i) $\operatorname{rank} H=2$ and $r \in\{2,3\}$,

(ii) $G \neq O$ and $r \in\{0,1\}$,

(iii) $G=O, r=1$, and $H \neq 0$.

Proof. The "if" part is obvious. Now we prove the "only if" part.

CASE 1. Suppose $G \neq 0$. Then, $\operatorname{det} G \neq 0$ from $G \in \mathscr{K}_{2}(\mathbf{F})$, and hence $V^{t} Y V=\operatorname{diag}(G$, $D_{1}, H^{t} G^{-1} H$ ) for some $V \in \mathrm{GL}_{n}(\mathbf{F})$. Using $Y \in \mathcal{M K}_{n}(\mathbf{F})$ yields either (i) or (ii).

CASE 2. Suppose $G=O$. Then,

$$
P^{t} Y P=\left[\begin{array}{ccc}
O & O & H \\
O & D_{1} & O \\
-H^{t} & O & O_{r \times r}
\end{array}\right]
$$

from (3.1). Using $Y \in \mathcal{M} K_{n}$ (F) yields either (i) or (iii).

The proof is completed.

LEMмA 3.3. Suppose $n \geq 4$ is an integer, $\mathbf{F}$ is any field except for $\{0,1\}, \mu \in \mathbf{F} \backslash\{0,1\}$, and $O \neq X \in \mathscr{K}_{n}(\mathbf{F})$. Then, $\operatorname{rank} X=2$ if and only if $X+Y \in \mathcal{M} K_{n}(\mathbf{F})$ or $\mu X+Y \in \mathcal{M} K_{n}(\mathbf{F})$ for any $Y \in M K_{n}(\mathbf{F})$.

Proof. We first prove the "if" part by the reduction absurdity. Assume that $\operatorname{rank} X \neq$ 2. Then, $\operatorname{rank} X \geq 4$ from $O \neq X \in \mathscr{K}_{n}(\mathbf{F})$, and hence there exist matrices $X_{1} \in \mathscr{K}_{n-4}(\mathbf{F})$ and $P \in \mathrm{GL}_{n}(\mathbf{F})$ such that $X=P^{t} \operatorname{diag}\left(J, J, X_{1}\right) P$ by (iii) in Section 1. Choosing $Y_{0}=$ $-P^{t} \operatorname{diag}\left(\mu J, J, X_{2}\right) P \in M K_{n}(\mathbf{F})$ yields that $X+Y_{0} \notin M K_{n}(\mathbf{F})$ and $\mu X+Y_{0} \notin M K_{n}(\mathbf{F})$, a contradiction. The proof of the "if" part is complete.

Now, we prove the "only if" part. For any $Y \in M K_{n}(\mathbf{F})$, by Lemma 3.1, we can assume that (3.1) holds for some $P \in \mathrm{GL}_{n}(\mathbf{F})$ and $G \in \mathscr{K}_{2}(\mathbf{F})$. Thus,

$$
\mu_{0} X+Y=P^{t}\left[\begin{array}{ccc}
G+\mu_{0} J & O & H \\
O & D_{1} & O \\
-H^{t} & O & O_{r \times r}
\end{array}\right] P, \quad \mu_{0} \in\{1, \mu\} .
$$

Obviously, $G+\mu_{1} J \neq O$ for some $\mu_{1} \in\{1, \mu\}$. This, together with $G+\mu_{1} J \in \mathscr{K}_{2}(\mathbf{F})$, implies that $\operatorname{det}\left(G+\mu_{1} J\right) \neq 0$. Letting

$$
U=\left[\begin{array}{ccc}
I_{2} & O & -\left(G+\mu_{1} J\right)^{-1} H \\
O & I & O \\
O & O & I_{r}
\end{array}\right] P
$$

and using (3.5), we have $\mu_{1} X+Y=U^{t} \operatorname{diag}\left(G+\mu_{1} J, D_{1}, H^{t}\left(G+\mu_{1} J\right)^{-1} H\right) U$. This, together with $Y \in M K_{n}(\mathbf{F})$ and Lemma 3.2, implies $\mu_{1} X+Y \in M K_{n}(\mathbf{F})$. The proof of the "only if" part is complete.

THEOREM 3.4. Suppose $n \geq 4$ is an integer and $\mathbf{F}$ is any field except for $\{0,1\}$. Then, $\phi: \mathscr{K}_{n}(\mathbf{F}) \rightarrow \mathscr{K}_{n}(\mathbf{F})$ is a linear bijective map preserving the max-rank if and only if $\phi$ is of the form (2.11) or (2.12). 
Proof. The proof of the sufficiency is omitted since it is easy. Now, we prove the necessity. Let $\mu \in \mathbf{F} \backslash\{0,1\}$. For arbitrary but fixed $A \in \mathscr{K}_{n}(\mathbf{F})$ with rank $A=2$, we have from Lemma 3.3 that either $A+B \in M K_{n}(\mathbf{F})$ or $\mu A+B \in M K_{n}(\mathbf{F})$ for any $B \in M K_{n}(\mathbf{F})$. Using the definition of $\phi$ yields either $\phi(A)+\phi(B) \in \mathcal{M} K_{n}(\mathbf{F})$ or $\mu \phi(A)+\phi(B) \in \mathcal{M} K_{n}(\mathbf{F})$ for any $\phi(B) \in M K_{n}(\mathbf{F})$. By Lemma 3.3, we have either $\phi(A)=O$ or $\operatorname{rank} \phi(A)=2$. This, together with the injectivity of $\phi$, implies that $\operatorname{rank} \phi(A)=2$. Therefore, $\phi$ is a linear bijective map on $\mathscr{K}_{n}(\mathbf{F})$ preserving rank 2. By Theorem 2.4, we have completed the proof.

Theorem 3.4 generalizes those corresponding results in $[4,8]$.

4. Linear maps preserving the determinant and adjoint. It should be pointed out that when $n$ is odd, all linear maps on $\mathscr{K}_{n}(\mathbf{F})$ preserve the determinant, since the determinant of any matrix in $\mathscr{K}_{n}(\mathbf{F})$ is equal to 0 , and the adjoint of an alternate matrix may be nonalternate. For these reasons, we have to restrict $n$ to be even in this section.

Now, we study the linear preservers of the determinant (resp., adjoint) on $\mathscr{K}_{n}(\mathbf{F})$ using Theorem 3.4, where $n \geq 4$ is even. We first investigate the following theorem which generalizes the corresponding result in [6].

TheOREM 4.1. Suppose $\mathbf{F}$ is any field except for $\{0,1\}$ and $n \geq 4$ is even. Then, $\phi$ : $\mathscr{K}_{n}(\mathbf{F}) \rightarrow \mathscr{K}_{n}(\mathbf{F})$ is a linear map preserving the determinant if and only if $\phi$ has one of the forms (2.11) or (2.12), where $\delta^{n}(\operatorname{det} Q)^{2}=1$.

Proof. The "if" part is obvious. Now, we prove the "only if" part. Firstly, we prove that $\phi$ is bijective. It suffices to show that $\phi$ is injective since $\phi$ is linear. In fact, if $\phi(A)=O$ for some $A \in \mathscr{K}_{n}(\mathbf{F})$, then there exists a matrix $B \in \mathscr{K}_{n}(\mathbf{F}) \operatorname{satisfying} \operatorname{det}(A+$ $B) \neq 0$ and

$$
\operatorname{rank} A+\operatorname{rank} B=n
$$

Thus, $\operatorname{det} B=\operatorname{det} \phi(B)=\operatorname{det}(\phi(A)+\phi(B))=\operatorname{det} \phi(A+B)=\operatorname{det}(A+B) \neq 0$. This, together with (4.1), implies $\operatorname{rank} A=0$, or equivalently, $A=O$. Therefore, $\phi$ is injective.

Secondly, noting that $n$ is even, it follows from $\operatorname{det} \phi(X)=\operatorname{det} X$ for all $X \in \mathscr{K}_{n}(\mathbf{F})$ that $\phi\left(M K_{n}(\mathbf{F})\right) \subset \mathcal{M} K_{n}(\mathbf{F})$ and $\phi\left(\mathscr{K}_{n}(\mathbf{F}) \backslash M K_{n}(\mathbf{F})\right) \subset \mathscr{K}_{n}(\mathbf{F}) \backslash M K_{n}(\mathbf{F})$.

Combining the above two aspects yields that $\phi$ is a linear bijective map on $\mathscr{K}_{n}(\mathbf{F})$ preserving the max-rank. By Theorem 3.4, $\phi$ has one of the forms (2.11) or (2.12). Choosing $X=\sum_{i=1}^{n / 2} J_{i}^{(n)}$ yields $\delta^{n}(\operatorname{det} Q)^{2}=1$. The proof is complete.

In [1], Chan et al. characterize all nonzero linear maps on $\mathcal{K}_{n}(\mathbf{F})$ preserving the adjoint when $n \geq 4$ is even and $\mathbf{F}$ is any infinite field of characteristic not 2. Now, we generalize the result to any field except for $\{0,1\}$.

THEOREM 4.2. Let $\mathbf{F}$ be a field except for $\{0,1\}$ and $n \geq 4$ even. Then, $\phi$ is a nonzero linear map on $\mathscr{K}_{n}(\mathbf{F})$ preserving the adjoint if and only if there exist $\lambda \in \mathbf{F}^{*}$ and $Q \in$ $\mathrm{GL}_{n}(\mathbf{F})$, which satisfy $\lambda^{n-2}=1$ and $Q^{-1}=\alpha Q^{t}$ for some $\alpha \in \mathbf{F}^{*}$, such that either

$$
\phi(X)=\lambda Q X Q^{-1} \quad \forall X \in \mathscr{K}_{n}(\mathbf{F})
$$


or

$$
n=4, \quad \phi(X)=\lambda Q X^{*} Q^{-1} \quad \forall X \in \mathscr{K}_{4}(\mathbf{F})
$$

where $X^{*}$ is defined by (1.3).

Proof. The "if" part is obvious. Now, we prove the "only if" part. Firstly, we prove

$$
\phi\left(\mathscr{K}_{n}(\mathbf{F}) \backslash \mathcal{M} \mathcal{K}_{n}(\mathbf{F})\right) \subseteq \mathscr{K}_{n}(\mathbf{F}) \backslash \mu \mathscr{K}_{n}(\mathbf{F}) .
$$

For any $X \in \mathscr{K}_{n}(\mathbf{F}) \backslash M K_{n}(\mathbf{F})$, by the fact $\left[A^{\text {ad }}\right]^{\text {ad }}=(\operatorname{det} A)^{n-2} A$ for all $A \in \mathscr{K}_{n}(\mathbf{F})$, we get $\left[\phi(X)^{\text {ad }}\right]^{\text {ad }}=(\operatorname{det} \phi(X))^{n-2} \phi(X)$ and $\phi\left(\left(X^{\mathrm{ad}}\right)^{\mathrm{ad}}\right)=\phi\left((\operatorname{det} X)^{n-2} X\right)=$ $(\operatorname{det} X)^{n-2} \phi(X)$. This, together with $\left[\phi(X)^{\mathrm{ad}}\right]^{\mathrm{ad}}=\phi\left(X^{\mathrm{ad}}\right)^{\mathrm{ad}}=\phi\left(\left(X^{\mathrm{ad}}\right)^{\mathrm{ad}}\right)$, gives

$$
(\operatorname{det} \phi(X))^{n-2} \phi(X)=(\operatorname{det} X)^{n-2} \phi(X) .
$$

Using $X \in \mathscr{K}_{n}(\mathbf{F}) \backslash M K_{n}(\mathbf{F})$ gives $(\operatorname{det} \phi(X))^{n-2} \phi(X)=O$, and hence $\phi(X) \in \mathscr{K}_{n}(\mathbf{F}) \backslash$ $M K_{n}(\mathbf{F})$. Therefore, (4.4) holds.

Secondly, we show that

$$
\phi\left(M K_{n}(\mathbf{F})\right) \subseteq M K_{n}(\mathbf{F})
$$

For any $A \in M K_{n}(\mathbf{F})$, it is clear that $\operatorname{det} A \neq 0$. If $\phi(A)=O$, taking $D \in \mathscr{K}_{n}(\mathbf{F})$ with $\operatorname{rank} D=2$ and $\phi(D) \neq O$ (note that the existence of $D$ follows from $\phi \neq 0$ ), we have from (4.5) that $(\operatorname{det}(A+\mu D))^{n-2}=(\operatorname{det} \phi(A+\mu D))^{n-2}=(\operatorname{det} \phi(\mu D))^{n-2}=$ $\mu^{n-2}(\operatorname{det} \phi(D))^{n-2}$ for all $\mu \in \mathbf{F}^{*}$. This, together with (4.4) and $D \in \mathscr{K}_{n}(\mathbf{F}) \backslash M \mathcal{K}_{n}(\mathbf{F})$, implies that $\operatorname{det}(A+\mu D)=0$ for all $\mu \in \mathbf{F}^{*}$. Applying $\mathbf{F} \neq\{0,1\}, \operatorname{rank} D=2$, and Lemma 3.3 to $X=D$ and $Y=A$, we obtain $A \notin M K_{n}(\mathbf{F})$, a contradiction. Therefore, $\phi(A) \neq O$. By (4.5), we have $(\operatorname{det} \phi(A))^{n-2}=(\operatorname{det} A)^{n-2} \neq 0$, or equivalently, $\operatorname{det} \phi(A) \neq 0$. Thus, $\phi(A) \in M K_{n}(\mathbf{F})$.

Thirdly, we prove that $\phi$ is bijective. It suffices to shows from the linearity of $\phi$ that $\phi$ is injective. This is proceeded by the reduction to absurdity. Suppose that $\phi(A)=O$ for some nonzero $A \in \mathscr{K}_{n}(\mathbf{F})$. Without loss of generality, we can assume that $A=\operatorname{diag}\left(J, A_{1}\right)$ for some $A_{1} \in \mathcal{K}_{n-2}(\mathbf{F})$. Denote $B=\operatorname{diag}(-J, J, \ldots, J)$. Then, $\operatorname{det} B \neq 0$ and $\operatorname{det}(A+B)=0$. Using (4.4) and (4.6) yields $\operatorname{det} \phi(B) \neq 0$ and $\operatorname{det} \phi(A+B)=0$, which contradicts $\phi(A)=O$.

Combining the above three aspects yields that $\phi$ is a linear bijective map on $\mathscr{K}_{n}(\mathbf{F})$ preserving the max-rank. By Theorem 3.4, $\phi$ has one of the forms (2.11) or (2.12). For $a \neq 0$, take $B_{a}=a J_{1}^{(n)}+\sum_{i=2}^{n / 2} J_{i}^{(n)}$, it easy to see that $B_{a}^{\text {ad }}=\left(a^{2}-a\right) J_{1}^{(n)}-a^{2} B_{a}$.

When $\phi$ is of the form (2.11), that is, $\phi(X)=\delta Q^{t} X Q$ for all $X \in \mathscr{K}_{n}(\mathbf{F})$. Let $Q Q^{t}=$ $Q_{1}=\left[q_{i j}\right]$, where $q_{i j} \in \mathbf{F}$. Note that $\phi(A) \phi(A)^{\text {ad }}=(\operatorname{det} \phi(A)) I_{n}$ for every $A \in \mathscr{K}_{n}(\mathbf{F})$. This, together with $f\left(B_{a}\right)^{\text {ad }}=f\left(B_{a}^{\text {ad }}\right)$, gives $Q_{1} B_{a}^{\text {ad }} Q_{1}=\delta^{n-2} \operatorname{det}\left(Q_{1}\right) B_{a}^{\text {ad }}$. Thus, in a similar argument to that in [1, Theorem 4], we see that $Q_{1} J_{1}^{(n)}=\delta^{n-2} J_{1}^{(n)} Q_{1}^{\text {ad }}$. In general, we have

$$
Q_{1}\left(E_{i j}-E_{i j}\right)=\delta^{n-2}\left(E_{i j}-E_{i j}\right) Q_{1}^{\text {ad }} \quad \forall i \neq j .
$$


Note that $Q_{1}$ is symmetric, by (4.7) we have

$$
q_{s i}=q_{s j}=0 \quad \forall i, j \neq s, \quad q_{i i} q_{j j}-q_{i j}^{2}=\delta^{n-2} \operatorname{det} Q_{1} .
$$

This gives $d \in \mathbf{F}^{*}$ with $d^{2}=\delta^{n-2} Q_{1}$ such that $Q_{1}=d I_{n}$ and $(d \delta)^{n-2}=1$. Hence, $\phi$ is of the first form in this theorem.

When $\phi$ is of the form (2.12), similarly, we obtain that $\phi$ is of the second form in this theorem.

REMARK 4.3. When $n=4$, [1, Theorem 4] shows that the map

$$
f:\left[\begin{array}{cccc}
0 & x_{12} & x_{13} & x_{14} \\
-x_{12} & 0 & x_{23} & x_{24} \\
-x_{13} & -x_{23} & 0 & x_{34} \\
-x_{14} & -x_{24} & -x_{34} & 0
\end{array}\right] \longmapsto\left[\begin{array}{cccc}
0 & x_{34} & x_{24} & x_{23} \\
-x_{34} & 0 & x_{14} & x_{13} \\
-x_{24} & -x_{14} & 0 & x_{12} \\
-x_{23} & -x_{13} & -x_{12} & 0
\end{array}\right]
$$

preserves adjoint matrices. However, it is easy to see that $f$ is one of the forms in Theorem 4.2 by choosing $Q=E_{14}+E_{23}+E_{32}+E_{41}$ and $\lambda=1$.

ACKNOWLEDGMENTS. The authors would like to thank the referees for their invaluable comments and suggestions on an earlier version of the paper. This work was supported by the NSF of China (no. 10271021), the NSF of Heilongjiang province (no. A01-07), and the fund of Heilongjiang Education Committee for Overseas Scholars (no. 1054HQ004).

\section{REFERENCES}

[1] G. H. Chan, M. H. Lim, and K.-K. Tan, Linear preservers on matrices, Linear Algebra Appl. 93 (1987), 67-80.

[2] N. Jacobson, Lectures in Abstract Algebra. Vol. II. Linear Algebra, D. Van Nostrand, New York, 1953.

[3] C.-K. Li and N.-K. Tsing, Linear preserver problems: a brief introduction and some special techniques, Linear Algebra Appl. 162-164 (1992), 217-235.

[4] M. H. Lim, Rank k preservers on second Grassmann product spaces, Malay. J. Sci. 3B (1975), 145-149.

[5] S. W. Liu and D. B. Zhao, Introduction to Linear Preserver Problems, Harbin Press, Harbin, 1997.

[6] M. Marcus and R. Westwick, Linear maps on skew symmetric matrices: the invariance of elementary symmetric functions, Pacific J. Math. 10 (1960), 917-924.

[7] Z.-X. Wan, Geometry of Matrices: in Memory of Professor L. K. Hua (1910-1985), World Scientific Publishing, Singapore, 1996.

[8] R. Westwick, Linear transformations on Grassmann spaces III, Linear and Multilinear Algebra 2 (1974/1975), 257-268.

Chongguang Cao: Department of Mathematics, Heilongjiang University, Harbin 150080, China E-mail address: caocg@h7ju.edu.cn

Xiaomin Tang: Department of Mathematics, Heilongjiang University, Harbin 150080, China E-mail address: tangxm@n 1 ju.edu.cn 


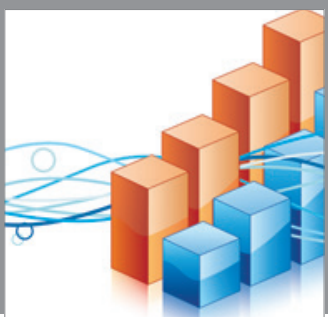

Advances in

Operations Research

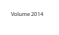

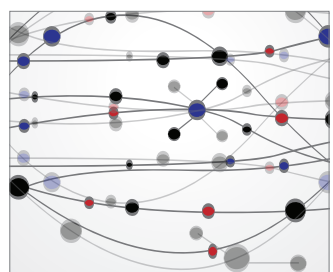

\section{The Scientific} World Journal


International Journal of

Mathematics and

Mathematical

Sciences
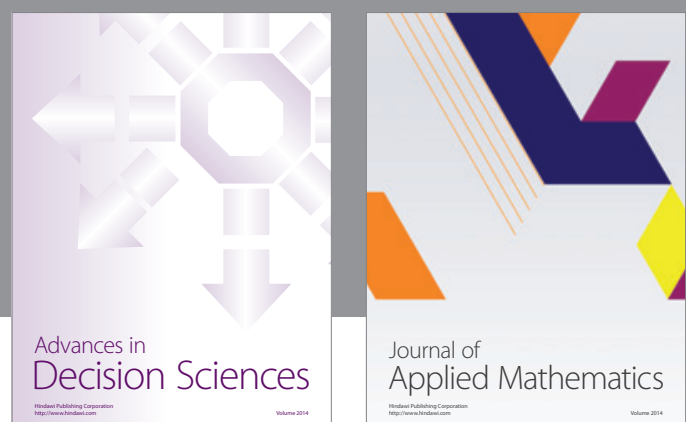

Journal of

Applied Mathematics
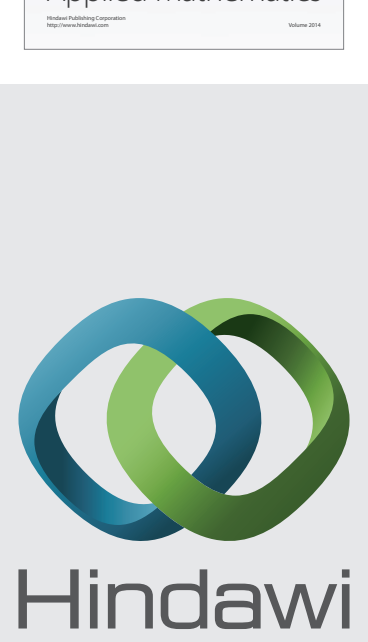

Submit your manuscripts at http://www.hindawi.com
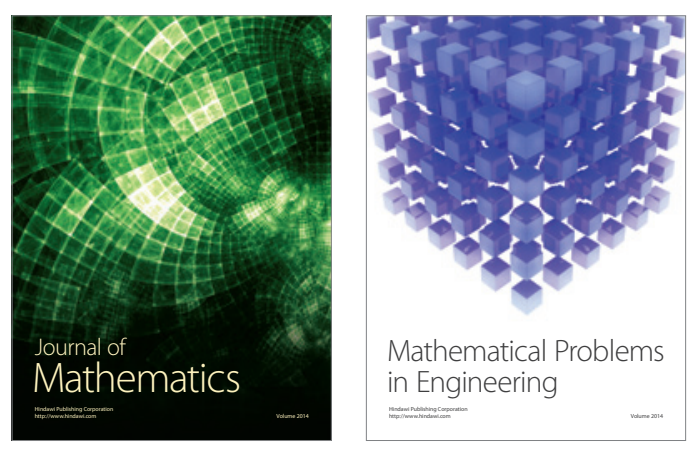

Mathematical Problems in Engineering


Journal of

Function Spaces


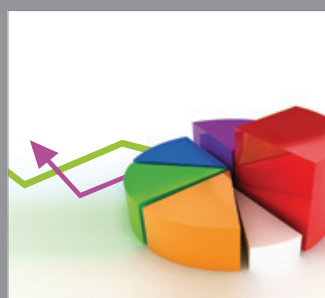

ournal of

Probability and Statistics

Promensencen
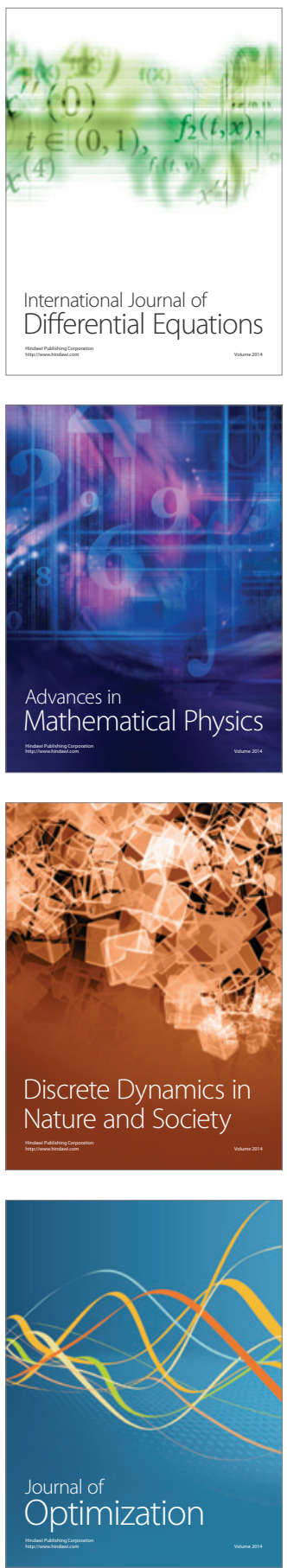